richtes! Weniger systematischer Einzelstoff! Dafür Vertiefung und Belebung des Unterrichtes, stärkere Hervorhebung der Bedeutung der Chemie für Natur, Kultur, Technik, Wirtschaft! Kein Unterricht ohne ,verbindliche" (nicht freiwillige) Utbungen! Für diese sind neue Unterrichtsstunden kaum nötig. ZweckmäBig wird $1 / 2-2 / 8$ der zur Verfügung stehenden Unterrichtszeit auf tbungen verwendet. Es schadet nichts, wenn der mündlicho Unterricht dadurch etwas eingeschränkt wird. Nur ungewöhnlich tüchtige Lehrer wissen wirklich Nützliches mit den vielen Stunden anzufangen, die jetrt z. B. an manchen preußischen Oberrealschulen für den mündlichen Chemie-Unterricht verwendet werden. Das Uherma $B$ des auf der Schule erworbenen chemischen Einzelwissens verleitet man. chen für Chemie völlig unbegabten Abiturienten der Realanstalten zum Studium der Chemie un l trägt zur Züchtung eines in jeder Hinsicht unerfreulichen Chemiker-Proletariates bei. Gerade den Lehrern der Realanstalten sei besondere Vorsicht beim Hinleiten ihrer Schüler zum Chemiestudium empfohlen; der für die Praxis brauchbare Chemiker hat andere Eigenschaften nötiger als eingetrichtertes Prüfungswissen!

Schulmänner bedauern, daß die Chemie in neuerer Zeit an den Realgymnasien, z. B. durch Fortfall der schriftlichen ReifeprüfungsArbeit seit der Reform von 1901 und durch Wegnehmen von Unterrichtsstunden für die Biologie, mehrfach gegenüber der Physik zurückgesetzt worden ist, sowie daß der Chemieunterricht in U II, von der viele Schüler mit dem „Einjährigen-Zeugnis" abgehen, nicht ausreicht (nur eine Wochenstunde in einem Halbjahr). Dem Wunsche, $\mathrm{daB}$ die Chemie an den Realanstalten in jeder Hinsicht als vollwertiges Hauptfach wie die Physik behandelt werde, muB man sich ansohließen.

(Schlub lolgt.)

\section{Beiträge zur Gewichtsanalyse. VI.}

Von L. W. Wringrar, Budapest.

(BohluB von 8. 188)

3. Bestimmung als Calciumcarbonat. Während bei der Bestimmung des Bariums und des Strontiums als Carbonat es sich sls nőtig erwies, zur Lösung Kaliumnitrat zu geben, um einen körnigen Niederschlag zu erhalten, erhält man aus einer Calciumchloridlösung auch ohne Kaliumnitratzusatz, bei richtigem Arbeiten, einen körnigen Niederschlag:

Die $100 \mathrm{ccm}$ betragende, höchstens $0,1 \mathrm{~g}$ Calcium entheltendo neutrale und ammoniumsalzfeie Lösung wind bis zum Aufkochen erhitzt, dann mit $10 \mathrm{ccm}, 10 \%$ iger" $\mathrm{N} \mathrm{a} \mathrm{-}$ triu m c a r b o natl ös ung versetzt; man erhält die Flüssigkeit noch einige Minuten in ruhigem Sieden. Geseiht wird am anderen Tage. Gewaschen wird mit $50 \mathrm{ccm}$ ausgekochtem, destilliertem Wasser, das mit Caloiumcarbonat gesättigt ist, oder mit $50 \mathrm{ccm}$ destilliertem Wasser, das I ccm Ammoniak und einige ccm Ammoniumcarbonatlösung enthält. Der anf $\operatorname{dem} W$ a $t$ t $\theta$ b a u 8 c b befindliche Niederschlag wird bei $132^{\circ}$ getrocknet.

$\mathrm{Zu}$ den Versu chen wurde dieselbe Calcinmohlorid. lös ung benutzt wie bisher. Es wurden Anteile ron 50, 10 and $1 \mathrm{ccm}$ ahgemessen und auf $100 \mathrm{com}$ verdünnt. Das Gewicht des Niederschlages sollte $256,77,51,35$ und $5,14 \mathrm{mg}$ betragen, während in Wirklichkeit folgende Zahlen erhalten wurden:

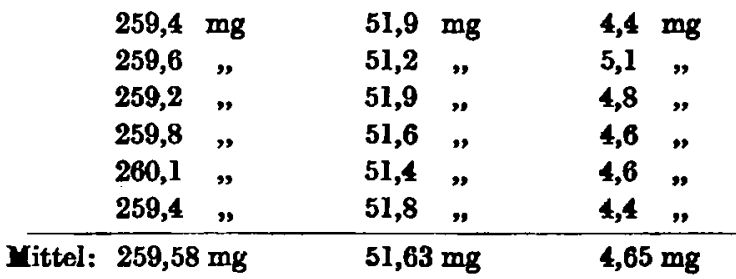

Die Verbegserungswerte berechnen sich ans diesen Zahlen:

$\begin{array}{cc}\text { Gewicht d. Nlederschlages } & \text { Verbesserungewert } \\ 0,25 \mathrm{~g} & -2,7 \mathrm{mg} \\ 0,20 " & -2,2 " \\ 0,15 " & -1,6 " \\ 0,10 " & -1,0 " \\ 0,05 " & -0,3 " \\ 0,02 " & +0,3 " \\ 0,01 " & +0,4 "\end{array}$

Beim Glühen mit der Gebläselampe betrug der Glührïckstand des bei $132^{\circ}$ getrockneten Niederschlages $55,63,55,70$ und 55,61, im Mittel $55,65 \%$.

Es wurden auch einige Versuche in Gegenwart f $\mathrm{rem} d \mathrm{er}$ $\mathrm{S}$ a $\mathrm{l}$ z ausgeführt, indem auf $100 \mathrm{com}$ Flüssigkeit $\left(=5 Q_{\mathrm{ccm}} \mathrm{Cal}\right.$. ciumchloridlösung) je $1,0 \mathrm{~g}$ des fremden Salzes genommen wurde:

$\begin{array}{cc}\text { In Gegenwart von } & \text { Getr. Niederschlag } \\ \mathrm{NH}, \mathrm{Cl} & 217,5 \mathrm{mg} \\ \mathrm{KCl} & 260,1 " \\ \mathrm{NaCl} & 259,4 " \\ \mathrm{KNO}_{3} & 260,0 " \\ \mathrm{KClO}_{3} & 259,6 " \\ \mathrm{Na}\left(\mathrm{C}_{2} \mathrm{H}_{3} \mathrm{O}_{2}\right) & 259,9 " \\ \mathrm{~K}_{2} \mathrm{CrO} & 261,1 "\end{array}$

Chlorammonium verhindert also das vollständige Ausfällen des Calciumcarbonates; in Gegenwart von Ammoniumsalzen wird man als Fällungsmittel ein Gemenge von Natronlauge und Natriumcarbonatlösung verwenden und bis zum Verschwinden des Am. moniakgeruches erwärmen. In Gegenwart von Chromsäure ist der Niederschlag chromathaltig und dementsprechend gelblich gefärbt.

$$
\text { Zusammonfassung. }
$$

Die gewichtsanalytischen Bestimmungsver. fahren des Calciums als Calciumoxalat, Cal. o i moxyd und $\mathrm{Calciumoarbonat}$ wurden auf ihre Gen a i g ke it ge prüf und die bei genauen Untersuchungen nótigen Verbesserungswerte ermittelt. Die Bestimmung als Calciumoxyd ist in Gegenwart von Sulfaten unrichtig. Am besten läBt sich das Calcium als $\mathrm{CaC}_{2} \mathrm{O}_{4}, \mathrm{H}_{2} \mathrm{O}$ bestimmen. [A. 82.]

\section{Kurze Notiz über die Umwandlung des Kalk- stickstoffs beim Lagern.}

\section{Von Dr. J. P. VAY ZYL.}

(Mitteilung ans dem agrikulturchemischen Institut der Oniveroitat Gottlogen. (IIIngeg. 15./8. 1918.)

Es ist allgemein bekannt, daß der Kalkstiokstoff verhältnismaBig leicht zur Umsetzung neigt, so daß bereits unter dem Einflu B der Feuchtigkeit und Kohlensäure der Luft dieses Düngemittel weitgehende Umwandlungen erleiden kann, wobei gewöhnlich ein beträchtlicher Teil des Stickstoffs überhaupt verlorengeht oder in Dicyandiamid, einen für die Pflanze als Gift zu bezeichnenden Körper, umgewandelt wird. Daher besteht in der Praxis auch der Brauch, den Kalkstickstoff möglichst bald nach der Anschaffung auf den Äckern zu verwenden, um also der Gefahr der Entwertung, die unter Umständen nach längerem Lagern fast vollständig werden kann, vorzubeugen.

Vor einigen Monaten ist unserem Institute cine Probe eines ungefăhr $21 \%$ Jahre alten Kalkstickstoffes von einem Gute in dem Landkreis Göttingen zugestellt worden. Untersucht wurde die zerpulverte Probe eines größeren Postens dieses Düngers, welcher zu ebener Frde in einer offenen Scheune lagerte und infolge langer, dann tödlicher Krankheit des Gutsherrn und späteren, fast vollständigen Wechsels des Personals wohl versehentlich nicht zur Anwendung gekommen war. Der Dünger soll bei der Lieferung etwa $19 \%$ Gesamtstickstoff enthalten haben; indessen gelang es uns unter den erwähnten Umständen leider nicht, genauere Einzelheiten über denselben zu erfahren.

Die Untersuchung im hiesigen Institut ergab einen noch immerhin auffallend hohen Gehalt an Gesamtstickstoff, nämlich 14,64\% (im Yittel von 3 Analysen). Um einigen Aufschlu B über die Art der Bindung des Stickstoffs zu erlangen, wurde eine größere Probe mit Wasser angerührt, ungeführ $30 \mathrm{Min}$. aufgekocht und die Flüssigkeit dann abgesaugt ${ }^{1}$ ). Der lufttrockene Rückstand enthielt nunmehr nur noch $1,39,1,23,1,34$, im Mittel 1,32\% N.; d. h. nur $9 \%$ des vorhandenen Stickstoffs wurde dem Kalkstickstoff durch diese Behandlung nicht entzogen. Aus dem Filtrat konnten in $100 \mathrm{~g}$ Kalkstickstoff rund $11 \mathrm{~g}$ umkrystallisierten, reinen Dicyandiamids, entsprechend also $7,3 \mathrm{~g} \mathrm{~N}$., d. h. $50 \%$ des vorhandenen N., gewonnen verden. (Die Reinheit des Produktes wurde durch die Analyse bewiesen. Der N-Gehalt desselben betrug $66,48 \%$. Der theoretische Wert ist 66,66\%.) Der Rest von rund $40 \%$ des Stickstoffs war wohl

1) $\mathrm{S}$ öll und $\mathrm{S}$ tützer, Ber. ohem. Gesell. Borlin 4\%, 4533 [1909]. 
überwiegend während der Behandlung mit kochendem Wasser als Ammoniak entwichen, was auch aus dem starken Ammoniakgeruch zu schließon war. Wenn nun auch hiernach dieser alte Kalkstickstoff dem durchsohnittlichen, frisch hergestellten Dünger in seinem Gesamt-N-Gehalt nur wenig nachsteht, und der N. zu mehr als $90 \%$ mit heißem Wasser ausziehbar ist, war doch noch die Möglichkeit vorhanden, daß ein erheblicher Teil seines N. in Dicyandiamid um. gewandelt sein konnte, wodurch unter Umständen seine Anwendung auf dem Acker bedenkliche Folgen hätte haben können. Es wurde daher nach der C a r o schen Methode, den Angaben von K a p p e n gemä $B^{2}$ ), die Bestimmung des Dicyandiamids vorgenommen. Dabei ergab sich für $1 \mathrm{~g}$ Kalkstickstoff in $\mathrm{mg}$ :

\begin{tabular}{lrrrr} 
& \multicolumn{1}{c}{1.} & \multicolumn{1}{c}{2.} & \multicolumn{1}{c}{. } & Mittol \\
Gesamtatickstoff . . & 130,6 & \multicolumn{1}{c}{132,7} & 130,7 & 131,3 \\
Cyanamid-N. . . & $\mathbf{9 9 , 6}$ & $\mathbf{9 7 , 5}$ & 101,5 & 99,5 \\
Dicyandiamid-N . . & 2,1 & 2,4 & 2,1 & 2,1
\end{tabular}

Beim viertelstündigen Erhitzen im Destillierkolben wurden außerdem $10 \mathrm{mg}$ Ammoniak-N. gefunden. Unser Kalkstickstoff war hiernach, trotz seines mehr als $21 / 2$ Jahre langen Lagerns, in merkwürdig frischem Zustande geblieben. Von dem Gesamt.N. der im Wasseraus zug gefunden wurde, war noch mehr als $75 \%$ in der ursprünglichen Bindungsform des Cyanamids vorhanden und nur $1,6 \%$ in Diojan. diamid umgewandelt. Der Rest des $\mathrm{N}$. dürfte wohl als Harnstoff usw., jedenfalls in einer für die Pflanze nicht schädlichen Form, vorhanden gewesen sein. Somit konnte hier der wohl seitene Fall festgestellt werden, daß der Kalkstickstoff nach langem Lagern ohne besondere Vorkehrungen doch nur einen verhältnismäßig sehr kleinen Bruchteil seines Wertes eingebüßt hatte. Interessant ist ein Vergleich mit dem vor einiger Zeit von $\mathbf{K}$ a $\mathbf{p}$ p e $\mathbf{n}$ untersuchten alten Kalkstickstoff, der nur noch $115,4 \mathrm{mg} \mathbf{N}$ auf $1 \mathrm{~g}$ Kalkstickstoff in dem Wassırauszug aufwies, wovon überhaupt nichts mehr als CyanamidN., dagegen über rund $73 \%$ als Dicyandiamid gebunden $\mathbf{w a r ^ { 2 }}$ ).

Wenn es nun auch nicht in dem Rahmen dieser Untersuchung lag, die Methoden der Dicyandiamidbestimmung einer Prüfung za untorziehen, so wurden doch mit dem übriggebliebenen kleinen Rest der Probe einige Bestimmungen des Dicyandiamidatickstoffs nach der Methode von $\mathrm{H}$ a ge $\mathbf{r}$ und $\mathrm{K}$ e $\mathbf{r} \mathrm{n}$ vorgenommen. Da der Wasserauszug wegen seines Gehaltes an Cyanamid sich hierfür nicht eignete, mußte ein alkoholischer Auszug hergestellt werden'). Im übrigen wurde wie bei $K$ a $p$ p e $n$ verfahren. Für $1 \mathrm{~g}$ des angewandten Kalkstickstoffs wurde in diesem Auszug gefunden: Dicyandiamid-N. nach $H$ a g e r 10,8,11,8, 11,0, im Mittel $11,2 \mathrm{mg}$.

Obwohl dieser Wert immerhin noch recht klein ist im Vergleich zu dem für Cyanamid-N. gefundenen, ist er doch ein Mehrfaches des C a r o schen. Da bei dem Mangel an weiterem, eigenem Analysonmaterial die Frage nach der Ursache dieses Unterschieds hier nicht erörtert werden kann, sei nur hervorgehoben, daB die hier mitgeteilten Ergrebnisse, soweit sie reichen, eine Bestätigung des $\mathbf{K}$ a p pe n schen Befunds bringen, und da $B$ auch hier die mutmaßliche Anwesenheit von Harnstoff neben der nur geringen Menge von Dicyandiamid eine wesentliche Rolle hätte spielen können.

[A. 103.]

\section{Uber ein neuartiges Prinzip für die Herstellung von Metallüberzügen.}

\section{Von M. U. Schoop, Zürich.}

(Eingez, 24,/7. 1918,

Wie ich anderorts mitgeteilt habe, sind von mir eine Reibe ron Verfahren vorgeschlagen und entwickelt worden, um Metalle zn schmelzen, zu zerstäuben und auf beliebige Objekte aufzuspritzen, wobei man von Blockmetall, Metallstaub oder -draht ausgehen k:nnn. Für die große Praxis sind ohne Frage die beiden letztgenannten Verfahren am wichtigsten, da hierbei, im Gegensatz zu der auch sonst umständlichen Methode mit flüssigem Metall, Gegenstände von belichiger Größe und Form (und an beliebigem Orte!) ohne weiteres mit Metallüberzüugen versehen werden können. Dieser in die Augen springende Vorzug kommt auch dem hier zu besprechenden neuen Arbeitsprinzip zu, welches Eleganz mit außerordentlicher Einfachheit verbindet und in dieser Hinsicht wohl kaum nooh zu übertreffen ist.

2) H. K a p pe n, Angew. Chem. 31, I, 31 [1918].

3) Angew. Chem. 31 I, 32 [1918]

4) $\mathrm{H}$ a g e r und $\mathrm{K}$ er $\mathrm{n}$, Angew. Chem. $29 \mathrm{I}, 312$ [1916].
Fin Blick auf die untenstehenden Abbildungen genügt, um ohne langatmige Erklürung za erkennen, wie die Sache gemeint ist. In eine Bunsenflamme wird das Ende eines Bleiröhrchens hineingehalten, welches an eine Kohlensäureflasche angeschlossen ist. Das im heißen Flammenkegel des Bunsenbrenners stetig abschmelzende Blei wird nun von dem, mittels des Röhrchenkanales zu. geleiteten Preßgases gefaßt, mehr oder weniger fein zerteilt und mit Wucht fortgeschleudert. Der erforderliche Druck des Preßgases hångt hierbei selbstrerstăndlich von der Rohrlänge und dem Kanalquerschnitt ab, ist jedoch durch einige Vorversuche leicht zu ermitteln. Das zerteilende und transportierende Medium ist, wie auch

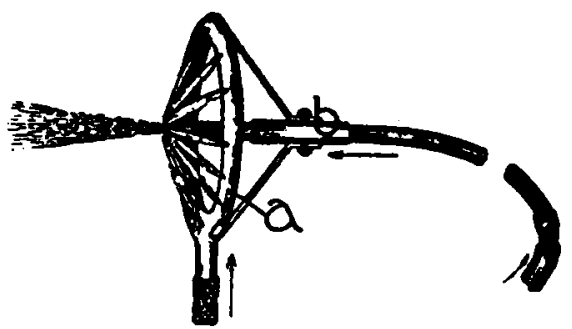

bei früheren Versuchen ăhnlicher Art, ein verdichtetes Gas; hingegen ist das neue Verfahren insofern grundsätzlich neu, als das Gas z entral zugeleitet wird, wogegen bei dem sogenannten Drahtspritzverfahren die Flammengase sowie der Zerstäuber- und Transportwind mit der Drabt peripherie in Berührung kommen, und zwar unter Bildung eines Strahlkegels von außerordentlich fein zerstäubtem Metall. Dieser feine Strahlkegel ist bei der obigen Versuchsanordnung nicht vorhanden; vielmehr wird das abschmelzende Metall in Form kleiner Tröpfchen, also ohne eigentliche Zerstäubung aufgespritzt, wobei ein überraschend gleichmäßiger und festhaftender Utberzug aus normalem, gesundem Blei entsteht. Die derart erhaltenen Bleiüberzüge sind, wie die mikroskopische Prüfung zeigt, wirklich rein, und ich neige daher zu der Auffassung, daß man mit der vorliegenden Erfindung dem Problem, die homogene Verbleiung, das Schmerzenskind der chemischen Großindustrie, vollgültig zu ersetzen, um einen túohtigen Schritt nähergekommen ist.

Die große Reinheit des Bleiüberzuges wird, wie naheliegend dann besonders gewährleistet, wenn man für den Zerstäuber- und

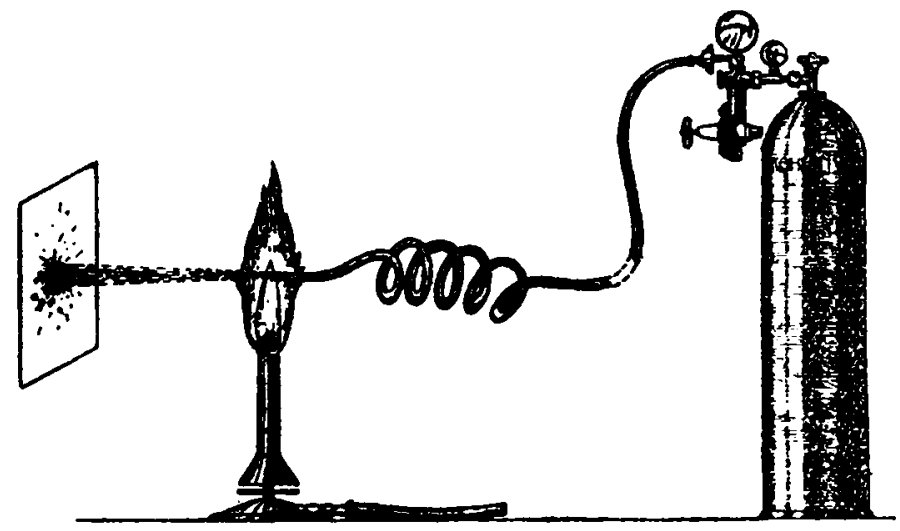

Transportwind anstatt Preßluft ein inertes oder reduzierendes Gas verwendet; dadurch wird jeder oxydierende Einfluß ausgeschaltet. Die Haftintensität wird unter dieser Voraussetzung so ausgezeichnet gut, da $B$ ein auf diese Weise verbleites Eisenblech beliebig oft hin und her gebogen werden kann, ohne daB ein Abblättern der Bleischicht zu befürchten ist. Will man ganz sicher gehen, so empfiehlt sioh, das bespritzte Blech v or dem Versuch von der Rückseite her zu erwärmen.

Der oben beschriebene Elementar- und Schulversuch läßt sich natürlich in mannigfacher Weise abändern; insbesondere kann man die zum Bohmelzen des Bleies notwendige Hitze vorteilhafter und wirtechaftlicher erzeugen, z. B. durch eine Anzahl von kreisförmig angeordneten Gasflämmohen oder durch elektrische Widerstandsorhitzung usw.

Ich habe als Ausführungsbeispiel Bleirohr gewählt, weil dasselbe verbältnismä Big leicht zu beschaffen ist, und der Schmclzpunkt des Bleies so niedrig ist, daß schon eine Bunsenflamme zum Abschmelzen genügt. Es steht aber nichts im Wege, nach dom oben kkizzierten „Röhrenprinzip“ mit jedem beliebigen Metall zu „metallisieren", ganz besondere, wenn man die Dienste der modernen und schmiegsamen Foe: Elektrizitat, in Anspruch nimmt. [A. 94.] 